\title{
ON SOME DIOPHANTINE PROBLEMS INVOLVING POWERS AND FACTORIALS
}

\author{
B. BRINDZA and P. ERDŐS
}

(Received 20 June 1989)

Communicated by J. H. Loxton

To the memory of Kurt Mahler

\begin{abstract}
In this paper the power values of the sum of factorials and a special diophantine problem related to the Ramanujan-Nagell equation are studied. The proofs are based on deep analytic results and Baker's method.
\end{abstract}

1980 Mathematics subject classification (Amer. Math. Soc.) (1985 Revision): 11 D 61.

\section{Power values of the sum of factorials}

Erdös visited Mahler a few days before his death in February 1988 and discussed with Mahler the paper, his last, on which Mahler had been working. Mahler had investigated the following question.

Let $k>1$ be an integer and consider those numbers of the form $\sum_{i=1}^{\infty} \varepsilon_{i} k^{i}$ where $\varepsilon_{i} \in\{0,1\}$ such that

$$
\sum_{i=1}^{\infty} \varepsilon_{i} k^{i}=x^{2}, \quad x \in \mathbb{Z}
$$

has infinitely many solutions (for $k=2$ this is of course trivial). Mahler conjectured that for $k \geq 5$ the equation (1) has only a finite number of solutions. A nontrivial solution, for $k=4$, is $1+7+7^{2}+7^{3}=20^{2}$.

The first author is a National Research Fellow, funded by the Australian Research Committee.

(C) 1991 Australian Mathematical Society 0263-6115/91 \$A2.00+0.00 
On seeing Mahler's question it seems natural to ask whether it is true that

$$
\sum_{i=1}^{\infty} \varepsilon_{i} i !=x^{z}, \quad \varepsilon_{i} \in\{0,1\}, \quad \sum_{i=1}^{\infty} \varepsilon_{i}<\infty
$$

has only finitely many solutions in $\varepsilon_{1}, \ldots, x, z \in \mathbb{Z}$ with $z>1$. But in this generality the question is hopeless. However, it is an old conjecture that

$$
1+n !=x^{2}
$$

has only the solutions $n=4,5,7$. We prove

THEOREM 1. For every positive integer $r$ there is an $n_{0}=n_{0}(r)$ such that none of the integers

$$
\sum_{i=1}^{r} n_{i} !, \quad n_{0}<n_{1}<\cdots<n_{r}
$$

are powerful; that is, each has a prime factor which divides $\sum_{i=1}^{\infty} n_{i} !$ to the first power.

Unfortunately, there seems to be no way to give an explicit value for $n_{0}(r)$.

Proof of Theorem 1. Denote by $p_{1}<\cdots<p_{l}$ the primes in the interval $\left(\frac{1}{2} n_{1}, n_{1}\right)$. Observe that

$$
\frac{1}{n_{1} !} \sum_{i=1}^{r} n_{i} !=0 \bmod \left[\prod_{j=1}^{l} p_{j}\right] ;
$$

otherwise one of the $p_{j}$ 's would divide $\sum_{i=1}^{r} n_{i} !$ to the first power only. From the known elementary inequality $\prod_{j=1}^{l} p_{j}>2^{1 / 2 n_{1}}$ we obtain

$$
\frac{1}{n_{1} !} \sum_{i=1}^{r} n_{i} !>2^{1 / 2 n_{1}}
$$

which easily implies

$$
n_{r}>n_{1}\left(1+\frac{c_{1}}{\log n_{1}}\right)
$$

where the constant $c_{1}$ depends only on $r$.

Now we must use a strong theorem on prime numbers for which there is no effective proof (though such a proof could be constructed in principle).

There is an absolute constant $c_{2}$ so that for large $n$ and $d>n^{3 / 4}$

$$
\pi(n+d)-\pi(n)>\frac{c_{2} d}{\log n}
$$

(See, for example, [2, page 167].) 
Applying this result we immediately have

$$
n_{2}<2 p_{1}<n_{1}+2 n_{1}^{3 / 4} \text {. }
$$

If $r=2$ then from (3) and (5)

$$
n_{1}+\frac{c_{1} n_{1}}{\log n_{1}}<n_{2}<n_{1}+2 n_{1}^{3 / 4}
$$

which is a contradiction for $n_{0}$ large enough.

In the sequel we may assume that $r \geq 3$. Let $2<s \leq r$ be the smallest index for which

$$
n_{s}>n_{1}+2 n_{1}^{3 / 4} \text { and } n_{s}-n_{s-1}>\left(n_{s-1}-n_{1}\right)\left(\log n_{1}\right)^{4}
$$

Such an $s$ does exist by (3). Moreover, by (3) and the minimality of $s$ we can assume that $n_{s-1}<n_{1}+n_{1}^{9 / 10}$.

Let $q_{1}, \ldots, q_{t}$ denote the primes between $n_{s-1} / 2$ and $\min \left(1 / 2 n_{s}, n_{1}\right)$. By (4), $t>\left(n_{s-1}-n_{1}\right)\left(\log n_{s-1}\right)$ (since $\log n_{1}$ and $\log n_{s-1}$ differ by $\log 2$ at most).

Now we show that

$$
\frac{1}{n_{1} !} \sum_{i=1}^{s-1} n !<\prod_{j=1}^{t} q_{j}
$$

Indeed,

$$
\frac{1}{n_{1} !} \sum_{i=1}^{s-1} n !<r n_{s-1}^{n_{s-1}-n_{1}}<n_{s-1}^{\left(n_{s-1}-n_{1}\right) \log n_{1}}<\left[\frac{n_{s-1}}{2}\right]^{t}<\prod_{j=1}^{t} q_{j}
$$

Hence there is a prime $q_{j}$ which does not divide $\left(1 / n_{1} !\right) \sum_{i=1}^{s-1} n !$.

On the other hand $n_{1}<n_{s-1}<2 q_{j}<n_{s}$ and $q_{j}<n_{1}$, and therefore $q_{j}$ divides $\sum_{i=1}^{r} n_{i}$ ! to the first power only, which completes the proof.

\section{The Ramanujan-Nagell equation and a related problem}

In the book of Erdös and Graham "Old and new problems and results in combinatorial number theory" it is asked "Is it true that the equation

$$
(p-1) !+a^{p-1}=p^{k}
$$

in positive integers $a, k, p$, with $p>2$ and prime, has only a finite number of solutions?" More than 150 years ago Liouville proved that

$$
(p-1) !+1=p^{k}
$$


has only two solutions: $p=3$ and $p=5$. For $a>1$, a non-trivial solution is given by $2 !+5^{2}=3^{3}$. It is interesting that if $p$ is not a prime then (6) has no solution, that is, the equation

$$
(n-1) !+a^{n-1}=n^{k}
$$

has no solution in positive integers $n, a, k$ with $n>2$ and not a prime. Indeed, if $n$ is a composite number then $n \mid(n-1) !$ and $n^{k}>(n-1) !$ implies $k>n-n / \log n$. Let $P$ be the largest prime factor of $n$. Then $(n-1)$ ! cannot be divisible by such a high power of $P$ except, possibly, if $P=2$. In this case, $n$ is a power of 2 and $a$ is even. Hence $2^{n-1}\left|a^{n-1}, 2^{n-1}\right| n^{k}$ but $2^{n-1}$ does not divide $(n-1)$ ! .

Returning to the equation (6), we prove

THEOREM 2. There exists an effectively computable absolute constant $C$ such that all solutions of the equation (6) satisfy

$$
\max \{p, a, k\}<C \text {. }
$$

This equation is a little eccentric but the proof of Theorem 2 is rather interesting. We shall show that for every solution

$$
\exp \left(C_{1} \frac{P}{\log p}\right)<k<C_{2} p^{3}
$$

where $C_{1}$ and $C_{2}$ are effectively computable absolute constants. Both the lower and upper bounds in (7) are proved by Baker's method and, surprisingly, the lower bound is much larger in $p$ than the upper one. The second part of (7) is a simple consequence of the following more general result on the Ramanujan-Nagell equation.

TheOREM 3. Let $D$ be a nonzero rational integer. Then all the solutions of the equation

$$
x^{2}+D=p^{k}
$$

in positive integers $x, p, k$ with $k, p>1$ satisfy

$$
\frac{k}{\log k}<C_{3}(p \log p+\log |D|) p \log p
$$

where $C_{3}$ is an effectively computable absolute constant.

This upper bound for $k$ is near to the best possible in $D$.

The proofs of Theorems 2 and 3 are based on the following deep results on linear forms in logarithms. 
Let $\alpha_{1}, \ldots, \alpha_{n}$ be nonzero algebraic numbers and let $A_{1}, \ldots, A_{n}$ be positive real numbers satisfying

$$
A_{j} \geq \max \left\{H\left(\alpha_{j}\right), e\right\}, \quad 1 \leq j \leq n
$$

where $H(\cdot)$ is the usual absolute height function.

Lemma 1 (Philippon and Waldschmidt [4]). Let $b_{1}, \ldots, b_{n}$ be rational integers such that

$$
\alpha_{1}^{b_{1}} \cdots \alpha_{n}^{b_{n}} \neq 1
$$

Let $B$ be a real number satisfying

$$
B \geq \max _{1 \leq i \leq n}\left|b_{i}\right| \text { and } B \geq e .
$$

Then $\left|\alpha_{1}^{b_{1}} \cdots \alpha_{n}^{b_{n}}-1\right|>\exp \left(C_{4} \log A_{1} \cdots \log A_{n} \log B\right)$ where $C_{4}$ is an effectively computable constant depending only on $n$ and on the degree of $\mathbb{Q}\left(\alpha_{1}, \ldots, \alpha_{n}\right)$ over $\mathbb{Q}$.

The following lemma is a special simple case of Yu's result for linear forms in the $p$-adic case.

LEMMA 2 (Yu [5]). Let $a_{1}, a_{2}$ be odd integers with $\left|a_{1}\right|\left|a_{2}\right|>1$ and let $b_{1}, b_{2}$ be rational integers such that $a_{1}^{b_{1}} a_{2}^{b_{2}} \neq 1$. Further, let $q>2$ be $a$ prime for which

$$
\left[Q\left(a_{1}^{1 / q}, a_{2}^{1 / q}\right): \mathbb{Q}\right]=q^{2} .
$$

Then

$$
\operatorname{ord}_{2}\left(a_{1}^{b_{1}} a_{2}^{b_{2}}-1\right)<C_{5} q^{6} \log \left|a_{1}\right| \log \left|a_{2}\right| \log \log \left|a_{1}\right| \log B
$$

where $B=\max \left\{2,\left|b_{1}\right|,\left|b_{2}\right|\right\}$ and $C_{5}$ is an effectively computable absolute constant.

Proof of Theorem 2. From (6) we immediately have $a>p, k \geq p$ and

$$
1 / 2(p-1) \leq \operatorname{ord}_{2}(p-1) !=\operatorname{ord}_{2}\left(p^{k} a^{-p}-1\right) .
$$

Preparatory to an application of Lemma 2, we prove the existence of a prime $q>2$ for which

$$
q<2 \log \log a \text { and }\left[\mathbb{Q}\left(p^{1 / q}, a^{1 / q}\right): \mathbb{Q}\right]=q^{2} .
$$

Indeed, there is a prime $2<q<2 \log \log a$ such that $a$ is not a $q$ th power, otherwise

$$
a \geq 3^{A} \text { with } A=\prod_{P<2 \log \log a} P \quad(P \text { prime })
$$


which is a contradiction. If $a^{1 / q}$ does not generate an extension of $Q\left(p^{1 / q}\right)$ of degree $q$ then, by Kummer theory, $a=p^{r} b^{q}$ where $0 \leq r<q, r \in \mathbb{Z}$ and $b \in \mathbb{Q}$. This is not possible since $a$ is not a $q$ th power and $(a, p)=1$. Thus we may apply Lemma 2 with an appropriate $q$, obtaining

$$
\operatorname{ord}_{2}\left(p^{k} a^{-p}-1\right)<c_{6} \log p \log a \log k(\log \log a)^{7}
$$

with $c_{6}=2^{6} c_{5}$. In the sequel $c_{7}, \ldots, c_{18}$ will denote effectively computable positive absolute constants. Comparing (10) with (9) we have

$$
\frac{1}{2}(p-1)^{2}<c_{6}(\log p)\left(\log a^{p-1}\right)(\log k)(\log \log a)^{7}<c_{7} k(\log k)^{8}(\log p)^{2}
$$

and that yields $p^{3 / 2}<c_{8} k$. Combining this inequality with (6) we have

$$
\left|a^{p-1} p^{-k}-1\right|=\frac{(p-1) !}{P^{k}}<\exp -c_{9} k \log p<\exp -c_{10} p \log a .
$$

However, from Lemma 1

$$
\left|a^{p-1} p^{-k}-1\right|>\exp -c_{11} \log a \log p \log k .
$$

The last two inequalities imply $\exp c_{12} \frac{p}{\log p}<k$.

To prove the second part of (7) we set $x=a^{(p-1) / 2}$ and $D=(p-1) !$. Then

$$
x^{2}+D=p^{k}
$$

and Theorem 3 gives $k>c_{13} p^{3}$ which completes the proof of Theorem 2 .

Proof of Theorem 3. We factorize equation (8) in the field $\mathbb{Q}(\sqrt{p})$ :

$$
\left((\sqrt{p})^{k}-x\right)\left((\sqrt{p})^{k}+x\right)=D \text {. }
$$

Let $\varepsilon$ be the fundamental unit for $\mathbb{Q}(\sqrt{p})$ with

$$
1<|\varepsilon|<\exp c_{14} p \log p \text {. }
$$

The norm of the factors $(\sqrt{p})^{k} \pm x$ is $D$ or $-D$. Hence the factors can be written in the form

$$
(\sqrt{p})^{k}+x=d_{1} \varepsilon^{t}, \quad(\sqrt{p})^{k}-x=d_{2} \varepsilon^{-t} \quad(t \in \mathbb{Z})
$$

where $d_{1}$ and $d_{2}$ are conjugate to one another (over $\mathbb{Q}$ ) and where we may assume that

$$
|\log | d_{i}||<c_{15} p \log p+\log |D|, \quad i=1,2
$$

(see for example [1, Lemma 3]). Let $\{1, \omega\}$ be an integral basis for $\mathbb{Q}(\sqrt{p})$ with $\omega \in\{\sqrt{p},(1+\sqrt{p}) / 2\}$ and $\varepsilon=u+v \omega$. Then

$$
|\varepsilon|>\frac{1}{2}\left(|\varepsilon|+\frac{1}{|\varepsilon|}\right) \geq|v \omega| \geq|\omega| \geq \frac{1}{2}(1+\sqrt{p}) \geq \frac{1}{2}(1+\sqrt{2})>1
$$


and from (11) and (12)

$$
|t|<c_{16}|t| \log |\varepsilon| \leq c_{16}\left(\log \left((\sqrt{p})^{k}+x\right)+|\log | d_{1} \mid\right)<c_{17} k \log p,
$$

under the assumption that $k>\max \{p, \log |D|\}$, for otherwise, Theorem 3 is proved. Obviously,

$$
d_{1} \varepsilon^{t}+d_{2} \varepsilon^{-t}=2(\sqrt{p})^{k} \text {. }
$$

Hence

$$
\Lambda=\left|2(\sqrt{p})^{k} d_{1}^{-1} \varepsilon^{-t}-1\right|<\frac{\left|(\sqrt{p})^{k}-x\right|}{\left|(\sqrt{p})^{k}+x\right|}<\frac{1}{(\sqrt{p})^{k}} .
$$

But from Lemma 1,

$$
\Lambda>\exp -c_{18}(\log p)(p \log p)(p \log p+\log |D|) \log k,
$$

which proves Theorem 3 .

REMARK. A $p$-adic version of a recent result of Mignotte and Waldschmidt [3] would lead to a sharper bound for $k$.

\section{References}

[1] K. Györy, 'Solutions of linear diophantine equations', Algebraic Integers of Bounded Norm, Ann. Univ. Scien. Budapest 22-23 (1980), 225-233.

[2] Y. Motohashi, Lectures on sieve methods and prime number theory, Springer, Berlin, 1989.

[3] M. Mignotte and M. Waldschmidt, 'Linear forms in two logarithms and Schneider's Method (II)', to appear.

[4] P. Philippon and M. Waldschmidt, 'Lower bounds for linear forms in logarithms', New Advances in Transcendence Theory, ed. A. Baker, Cambridge Univ. Press, 1988, pp. 280-313.

[5] $\mathrm{K} . \mathrm{Yu}$, 'Linear forms in logarithms in the $p$-adic case', New Advances in Transcendence Theory, ed. A. Baker, Cambridge Univ. Press, Cambridge, 1988, pp. 411-434.

Mathematical Institute

Kossuth Lajos University

4010 Debrecen

Hungary 